\title{
Master-slave control for prosthetic hands based on cybleglove
}

\author{
Wen $\mathrm{Ni}^{1,2}$, Liang Tang ${ }^{1,2}$ and Qunming $\mathrm{Li}^{1,2, a}$ \\ ${ }^{1}$ State key laboratory of High-Performance Complex Manufacturing; \\ ${ }^{2}$ School of Mechanical \& Electrical Engineering, Central South University, 410083 Changsha, China
}

\begin{abstract}
To achieve a human-like grasp for prosthetic hands, this paper proposes a masterslave control system for the prosthetic hands based on the Cybleglove II. The movement characteristics of human finger joints were acquired via the data glove, and analysed by the LabVIEW real-time control system simultaneously. Then the data were used to control the prosthetic hand. A series of experiments were conducted on the master-slave control system, whose result verifies the effectiveness of the master-slave control method.
\end{abstract}

Keywords: human-like; master-slave control; prosthetic hand; data glove.

\section{Introduction}

The control system in a prosthetic hand is thought to be the key factor in designing an automatically grasping system. With the emergence of advanced control technologies, many researchers have been pursuing a design of a high-performance control system for prosthetic hands for years but with limited success. Our brain can control our hand to grasp what we want perfectly. However, it is very difficult for a prosthetic hand, which cannot get the information of our brain to grasp the objects, to be controlled as a human-like prosthetic hand.

In the last two decades, the grasping research of prosthetic hands has drawn much attention of the control engineering community. A part of these researchers focuses on the control of prosthetic hands based on Electromyography (EMG) signals [1-8]. Some researchers [9-10] propose a real-time EMG pattern cognition approach by using wavelet transform. The patterns of motion have been identified by EMG. In addition, an EMG signal is a complicated signal, which can be used to control the prosthetic hand to grasp objects online. However, EMG signal produce noise while being traveled through different tissues. Moreover, the EMG detector collects signals from different motor units at a time, which may cause the interaction among different signals [11].

This paper presents an approach to control the prosthetic hand by using Cybleglove. The motion characteristic of finger joints were acquired via the Cybleglove, and then analyzed by the LabVIEW real-time control system, and the master-slave control system controls the prosthetic hand to grasp the object according to the analyzed motion data of human hands. In addition, the simulation analysis of the master-slave system is carried out, and the control performance of the control system is also examined by a series of experiments.

\footnotetext{
a Corresponding author : liqm@mail.csu.edu.cn
} 


\section{Description of the testbed}

The testbed is shown in Figure 1(a), which includes a prosthetic hand, the CyberGlove II, a PC and an entire NI LabVIEW real-time system.
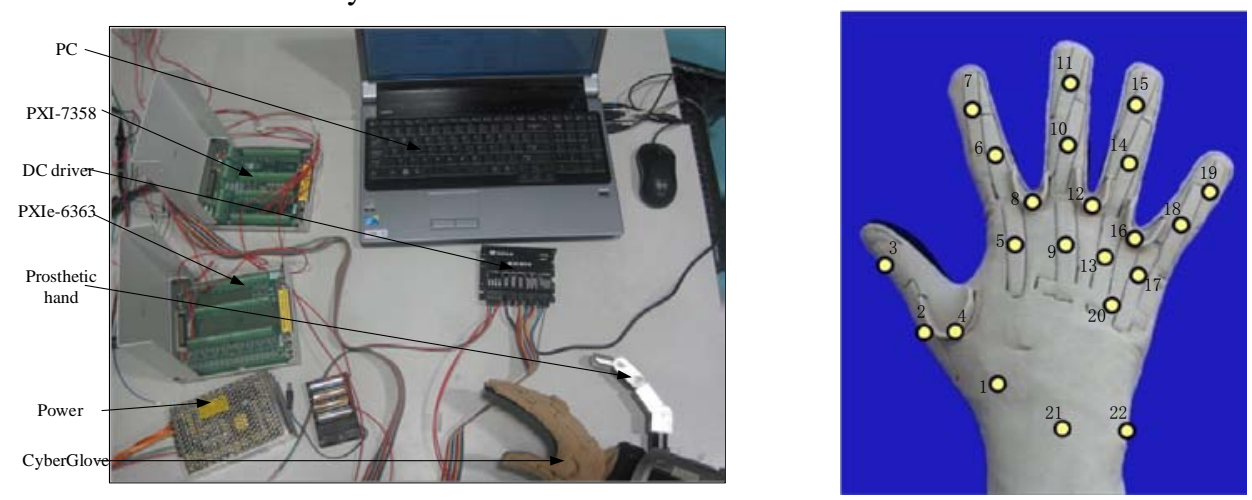

Figure 1. (a) The testbed; (b) Cyberglove II with 22 sensors.

The data glove shown in Figure 1 (b) is used in the experiments described in this paper. The Cyberglove II is an advanced data glove designed specifically for the recognition of signal information. It uses the proprietary resistive bend sensing technology to transform finger motion data into real-time diagonal joint angle data accurately. For this study, the required movement data were obtained by a PC and then sent to the LabVIEW real-time system simultaneously, and the data of 22 Cyberglove sensors were recorded with the sampling frequency of $50 \mathrm{~Hz}$.

\section{Master-slave control scheme}

There are two key factors in designing a master-slave control system for a prosthetic hand. One is the motion mapping relation between a human hand and a prosthetic hand, and the other is how to control the prosthetic hand to track the movement of a human hand.

We presented a method of motion mapping based on joint mapping and the fingertip position, and established a mathematical model of a prosthetic hand. The selected coordinate system is shown in Figure 2. Where $a_{1} a_{2}$ and $a_{3}$ are the length parameters of the three joints, $\beta_{1} \beta_{2}$ and $\beta_{3}$ are the angle of three joints.

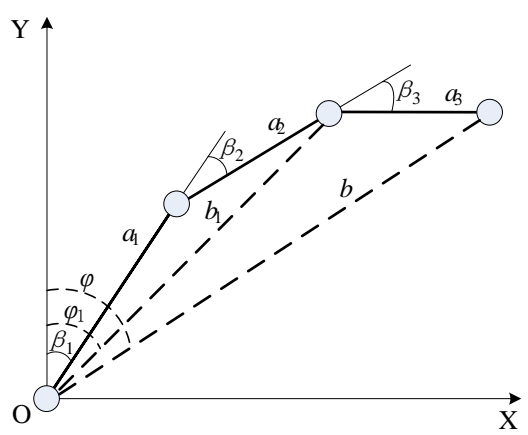

Figure 2. The selected coordinate system for the mathematical model.

Then we can obtain the angle of joint during grasping: 


$$
\begin{gathered}
\varphi_{1}=\beta_{1}+a \cos \left(\frac{a_{1}+a_{2} \cos \left(\beta_{2}\right)}{\sqrt{a_{1}^{2}+a_{2}^{2}+2 \cdot a_{1} a_{2} \cos \left(\beta_{2}\right)}}\right) \\
\varphi=\varphi_{1}+a \cos \left(\frac{b_{1}+a_{3} \cos \left(\beta_{3}\right)}{b_{1}^{2}+a_{3}^{2}+2 b_{1} a_{3} \cos \left(\beta_{3}\right)}\right)
\end{gathered}
$$

In addition, because the size of a prosthetic hand is larger than that of human hand, we get the fingertip position of the prosthetic hand by transition parameters in the Eq.3.

$$
\left\{\begin{array}{l}
x=a_{1} \sin \beta_{1}+a_{2} \sin \left(\beta_{1}+\beta_{2}\right)+a_{3} \sin \left(\beta_{1}+\beta_{2}+\beta_{3}\right)=b \sin \varphi \\
y=a_{1} \cos \beta_{1}+a_{2} \cos \left(\beta_{1}+\beta_{2}\right)+a_{3} \cos \left(\beta_{1}+\beta_{2}+\beta_{3}\right)=b \cos \varphi
\end{array}\right.
$$

According to the structural features of human hand and prosthetic hands, a master-slave control scheme based on the data glove was constructed in this paper, and is shown in Figure 3.

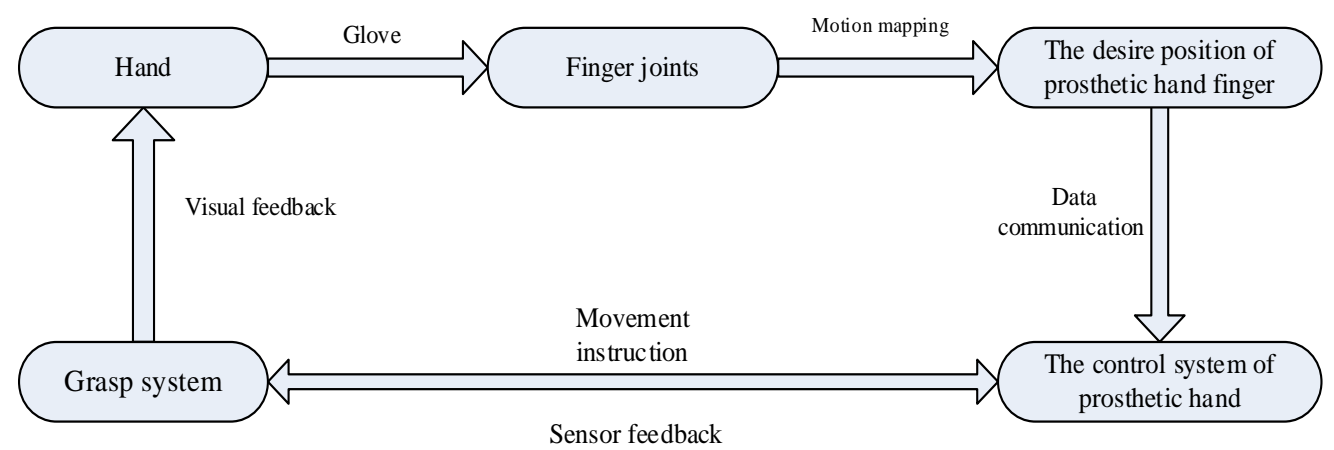

Figure 3. The structure of master-slave control scheme.

The data glove acquires the movement of human's finger joints, and then the data was analyzed by the LabVIEW real-time control system to obtain the corresponding movement of the prosthetic hand and human hand joints. Once the data glove obtains the grasp information, the real-time control system controls the prosthetic hand to grasp the object simultaneously.

The master-slave control system consists of three parts: the acquisition system, the prosthetic hand driving system and the sensor feedback system. The flow chart of master-slave control system is shown in Figure 4.

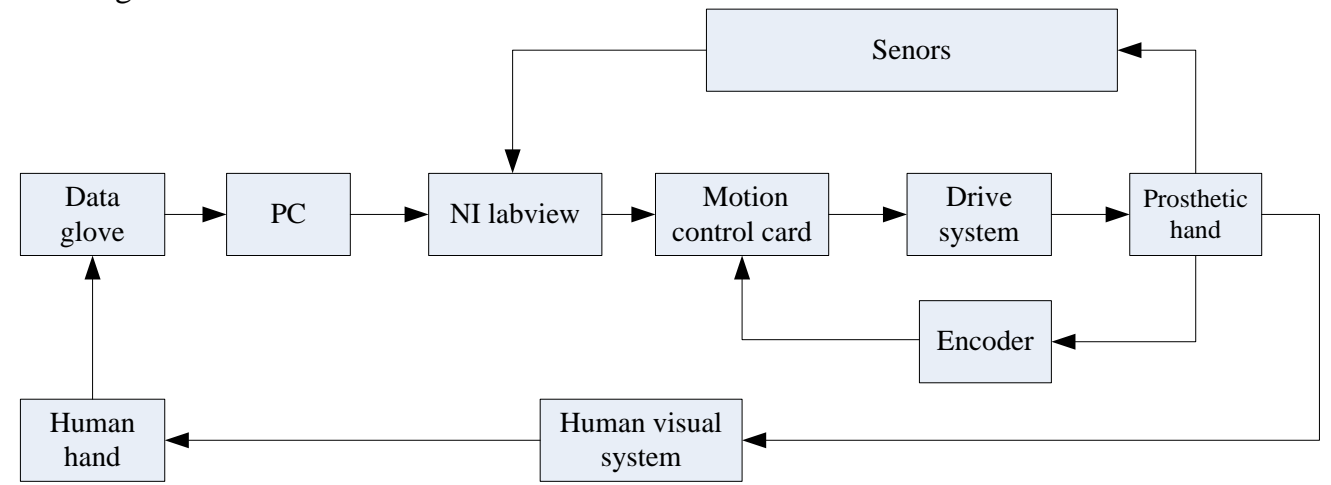

Figure 4. Flow chart the master-slave control system. 


\section{Experiments and analyses}

Experiments were carried out on index finger in order to measure the control error of the prosthetic hand. The deviation between the actual position and desire position was denoted as $\varepsilon$. The experiment results are shown in Figure 4.

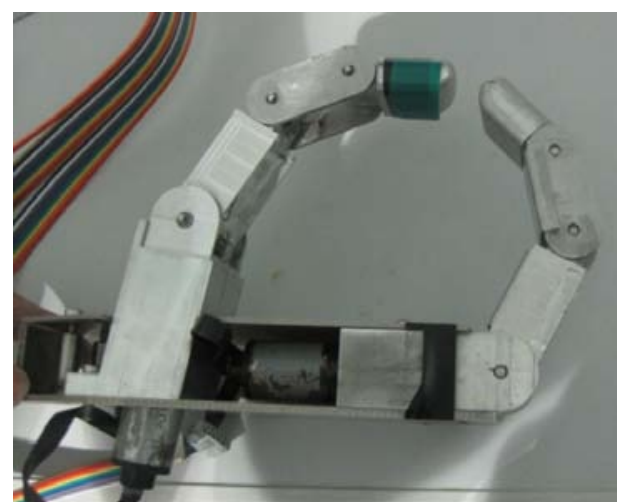

(a)

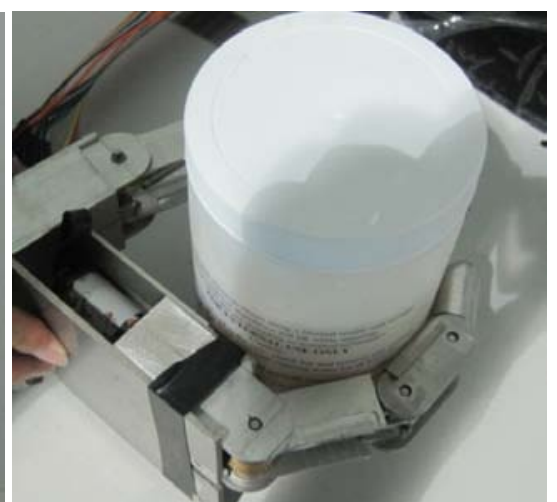

(b)

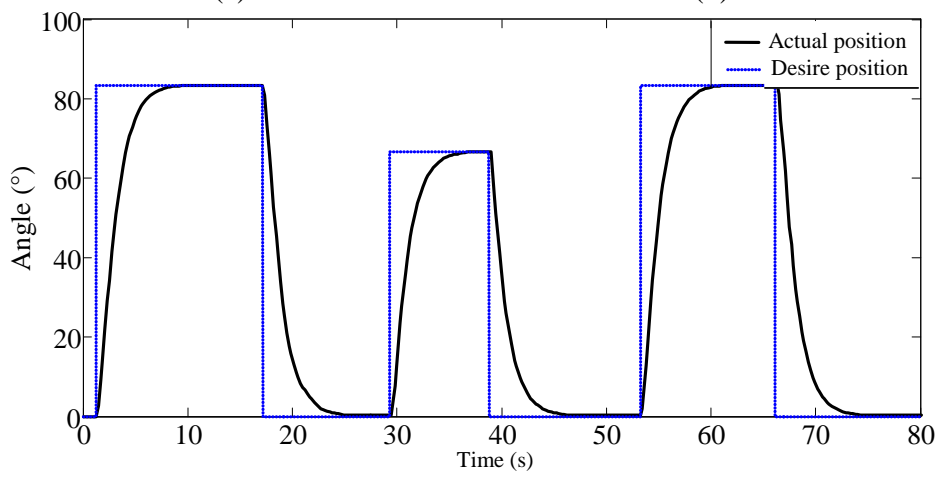

(c)

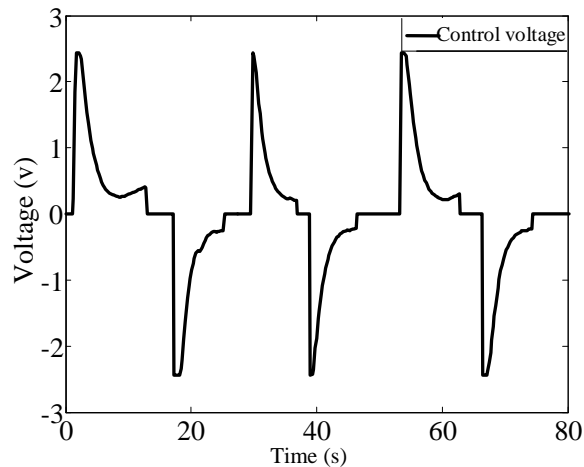

(d)

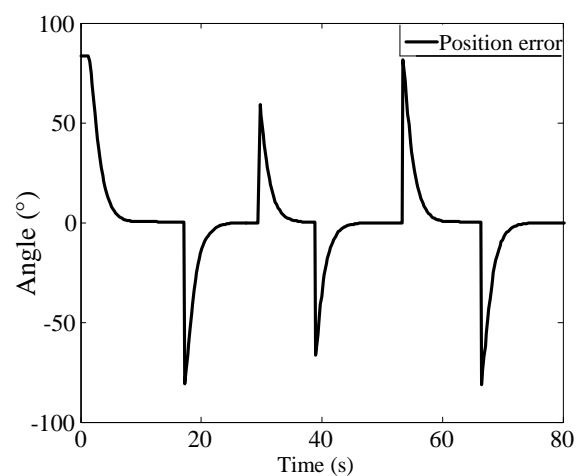

(e)

Figure 5. The experiment result; (a) and (b) the prosthetic hand grasping the objects; (c) the control error; (d) the control voltage; (e) the position error.

From Figure 4 (c), we can see that while the prosthetic hand tries to grasp the objects, it cannot response as fast as human hand. However, when the prosthetic hand stops grasping the object, it can also be seen that its last position are almost the same. This experiment result proves that the prosthetic hand performs well during grasping.

Then the experiments were conducted on the master-slave control system. The angle values of finger's three joints were acquired by the data acquisition glove system, and the data were sent to the 
controller to achieve a human-like grasp of the prosthetic hand. The experiments were conducted as follows: the data glove was operated by the experimenter, and the data of movement of three joints were acquired by the data glove; then the data were used to control the prosthetic hand online, when the experimenter tries to grasp the object, the data were analyzed by the real-time control system. The first set of experiments was carried out on the data glove and master-slave control system of the prosthetic without grasping an object.

The first set of experiments was conducted as follows: the data glove was fitted on the experimenter's hand; when the experimenter tried to grasp the object, the data of the whole movement process of the hand were recorded simultaneously, i.e., the experimenter finished the grasping process with a visual feedback system by human sensing; then the movement data were used in the masterslave control of the prosthetic hand to imitate the human hand's movement. The result of index finger is shown in Figure 6.

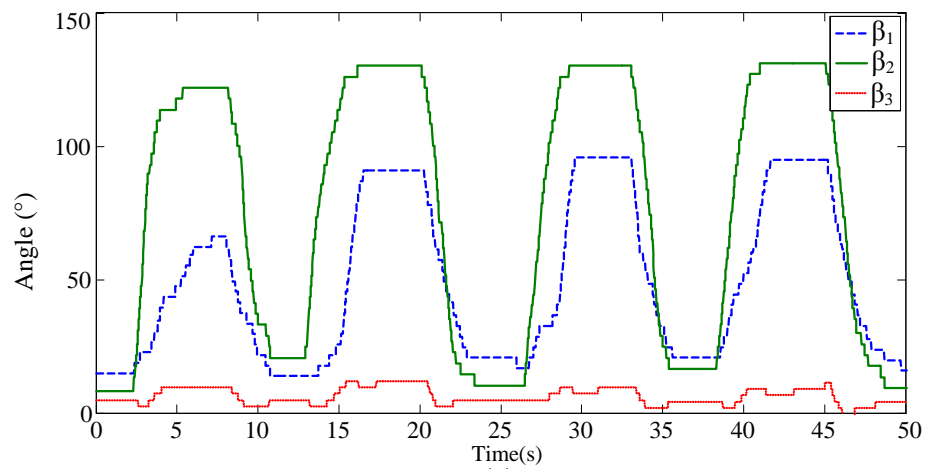

(a)

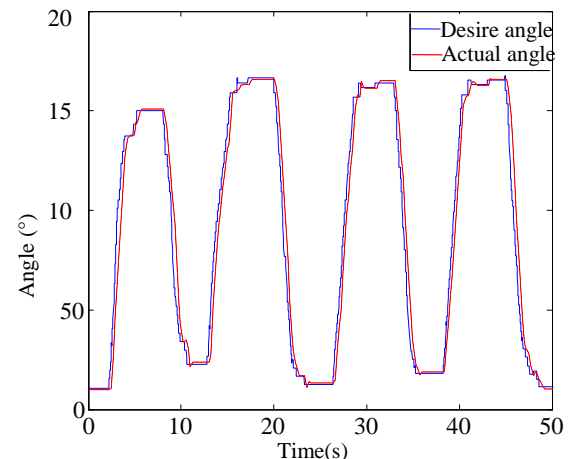

(b)

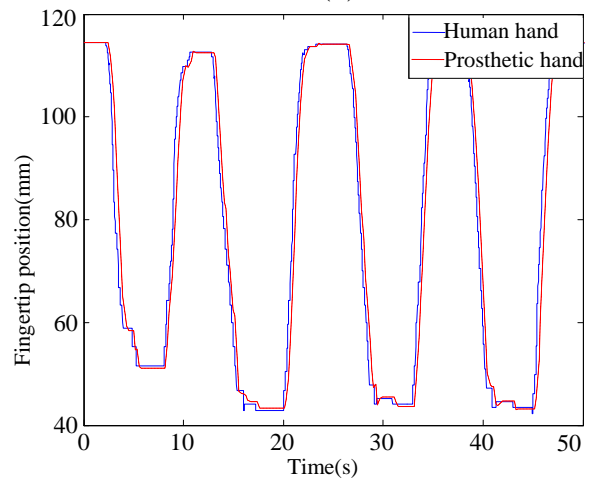

(d)

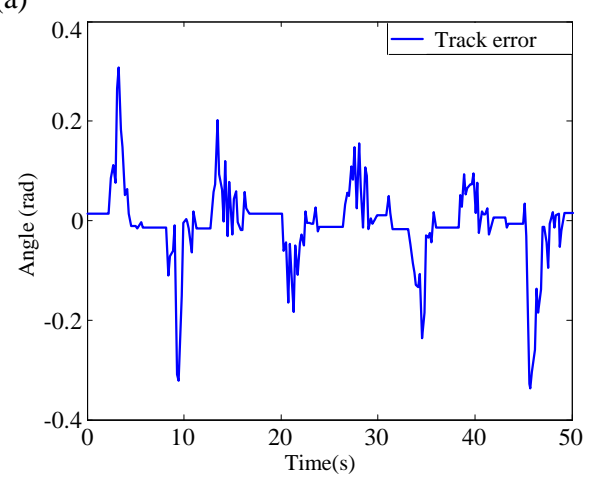

(c)

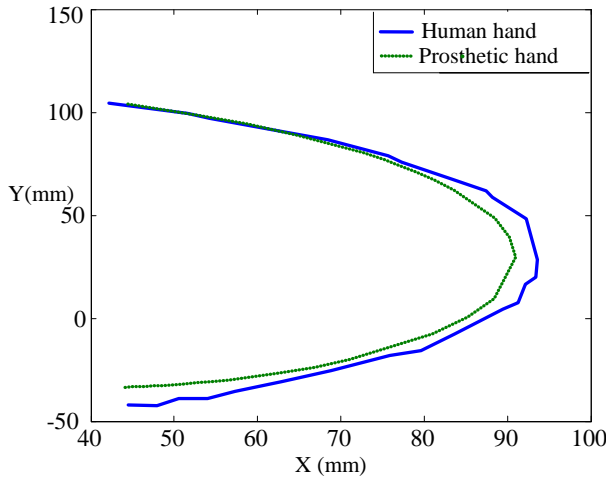

(e)

Figure 6. The experiment result with the prosthetic hand grasping nothing. (a) angels of three joints; (b) the motion mapping of angle; (c) the track error; (d) the fingertip position; (e) the trajectories of the prosthetic hand and the human hand. 
From the experiment results in Figure 6(c), (d) and (e), we can see that the track error ranges from $-0.4 \mathrm{rad}$ to $0.4 \mathrm{rad}$, but the fingertip positions of the prosthetic hand and human hand match well. In addition, we can also see that the movement trajectories of human hand and prosthetic hand were almost the same. This result proves that the master-slave control is useful.

In the second set of experiments, we conducted the experiments as the prosthetic hand trying to grasp the object like a human hand. The experimenter tries to grasp the cup, and then the fingers' movement data were used to control the prosthetic hand to track the human hand movement. The experiment results of index finger are shown in Figure 7.

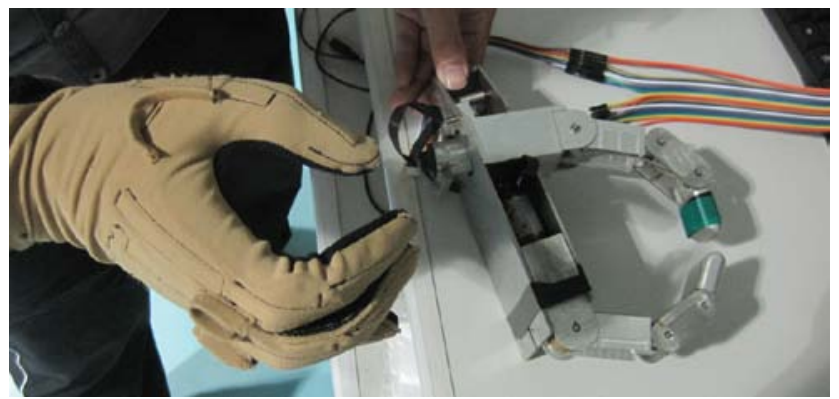

(a)

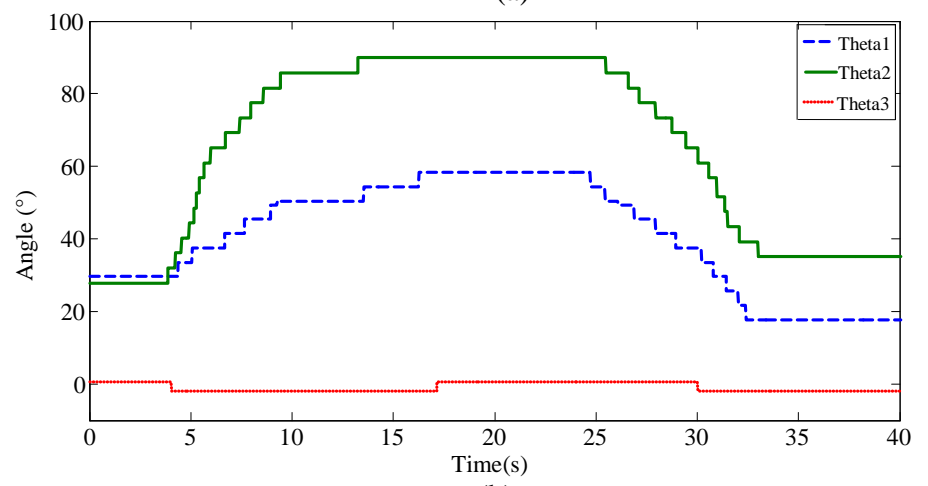

(b)

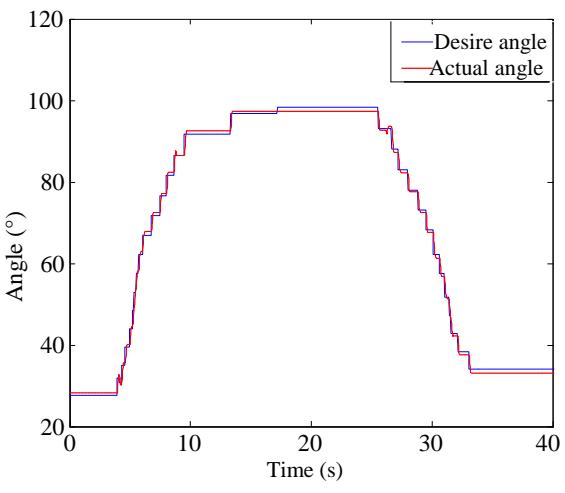

(c)

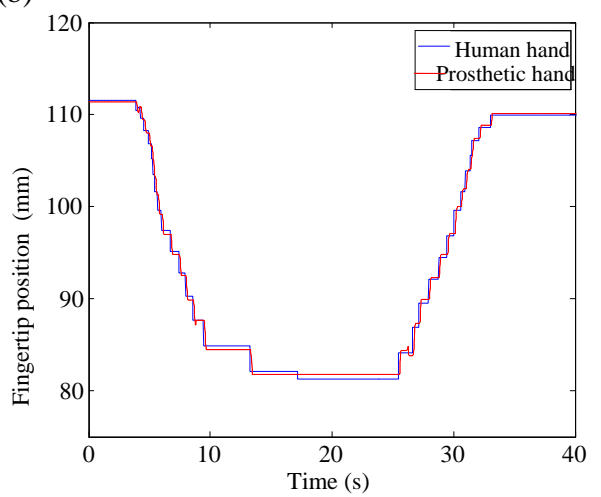

(d) 


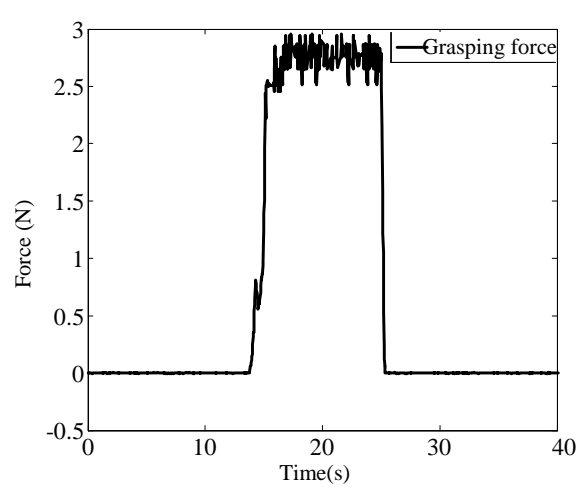

(e)

Figure 7. Experiment results (a) the experiment results; (b) angles of three joints; (c) angle of the finger; (d) position of fingertip; (e) grasping force.

From Figure 6, we can see that the master-slave control has good performance during the grasping , and that the prosthetic hand can grasp the object in real time. This proves that our master-slave control system is useful. From Figure (c) and (d), we can find that during the grasping of the prosthetic hand, the angles of joint movement and the position of the human hand which are acquired by the data glove match well with actual values. This proves the motion mapping and control is reasonable. In addition, when the prosthetic hand grasps the object, the average grasp force is about $2.75 \mathrm{~N}$ and the maximal and minimum grasping force are $2.5 \mathrm{~N}$ and $2.9 \mathrm{~N}$ respectively, which proves that the master-slave control system can control the grasping to be like human.

\section{Conclusions}

A master-slave control scheme was proposed base on CyberGlove II. Then experiments were conducted to exam the performance of the control system under a series of different conditions, whose results demonstrate the effectiveness of the master-slave control system. However, it should be noticed that there are still control errors in the use of the master-slave control system. This may because the accuracy of control system. The future work will be concentrated on improve the accuracy of master-slave control system.

\section{Acknowledgment}

In this paper, the research was sponsored by the grants from National Basic Research Program 973 of China (Grant No. 2011CB013302)

\section{References}

1. C. Castellini and P. van der Smagt, "Surface EMG in advanced hand prosthetics," Biological cybernetics, vol. 100, pp. 35-47, (2009).

2. K. Englehart and B. Hudgins, "A robust, real-time control scheme for multifunction myoelectric control," Biomedical Engineering, IEEE transactions on bio-medical engineering, vol. 50, pp. 848-854, (2003).

3. X. Chen and Z. J. Wang, "Pattern recognition of number gestures based on a wireless surface EMG system," Biomedical Signal Processing and Control, vol. 8, pp. 184-192, (2013).

4. R. Merletti and H. Hermens, "Detection and conditioning of the surface EMG signal," Electromyography: physiology, engineering, and noninvasive applications, pp. 107-131, (2004). 
5. J. Zhao, Z. Xie, L. Jiang, H. Cai, H. Liu, and G. Hirzinger, "EMG control for a five-fingered prosthetic hand based on wavelet transform and autoregressive model," in Mechatronics and Automation, Proceedings of the 2006 IEEE International Conference on, pp. 1097-1102. (2006).

6. P. Shenoy, K. J. Miller, B. Crawford, and R. P. Rao, "Online electromyographic control of a robotic prosthesis," IEEE Transactions on Biomedical Engineering, vol. 55, pp. 1128-1135, (2008).

7. K. Xing, P. Yang, J. Huang, Y. Wang, and Q. Zhu, "A Real-Time EMG Pattern Recognition Method for Virtual Myoelectric Hand Control," Neurocomputing, (2014).

8. M. B. I. Reaz, M. S. Hussain, F. Mohd-Yasin, "Techniques of EMG signal analysis: detection, processing, classification and applications (Correction) " Biological Procedures Online, pp. 163163. (2005).

9. J. U. Chu, I. Moon, Y.-J. Lee, S.-K. Kim and M.-S. Mun, "A supervised feature-projection-based real-time EMG pattern recognition for multifunction myoelectric hand control," IEEE/ASME Transactions on Mechatronics, vol. 12, pp. 282-290, (2007).

10. Z. Liu, Z. Luo, "Hand motion pattern classifier based on EMG using wavelet packet transform and LVQ neural networks," IEEE International Symposium on It in Medicine and Education. pp. 28-32. (2008).

11. S. Shahid, Higher Order Statistics Techniques Applied to EMG Signal Analysis and Characterization. Ph.D. thesis, University of Limerick. Ireland, (2004). 